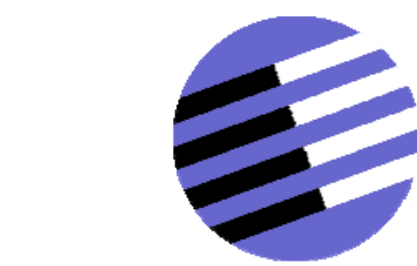

GOVERNANCE AND THE EFFICIENCY

OF ECONOMIC SYSTEMS

GESY

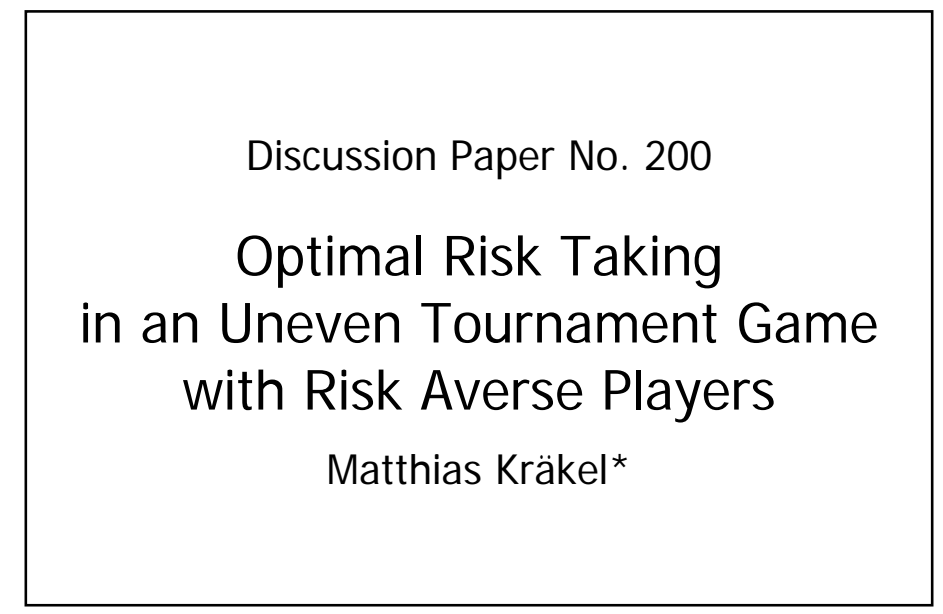

April 2007

* Matthias Kräkel, Department of Economics, BWL II, University of Bonn, Adenauerallee 24-42, D-53113 Bonn, Germany. Tel: +49-228-739211, Fax: +49-228-739210, m.kraekel@uni-bonn.de

Financial support from the Deutsche Forschungsgemeinschaft through SFB/TR 15 is gratefully acknowledged. 


\title{
Optimal Risk Taking in an Uneven Tournament Game with Risk Averse Players*
}

\author{
Matthias Kräkel, University of Bonn**
}

We analyze the optimal choice of risk in a two-stage tournament game between two players that have different concave utility functions. At the first stage, both players simultaneously choose risk. At the second stage, both observe overall risk and simultaneously decide on effort or investment. The results show that those two effects which mainly determine risk taking - an effort effect and a likelihood effect - are strictly interrelated. This finding sharply contrasts with existing results on risk taking in tournament games with symmetric equilibrium efforts where such linkage can never arise. Hence, previous findings based on symmetry at the effort stage turn out to be nongeneric.

Key words: asymmetric equilibria, rank-order tournaments, risk taking.

JEL classification: C72, J3, L1, M5

* I would like to thank Oliver Gürtler for helpful comments. Financial support by the Deutsche Forschungsgemeinschaft (DFG), grants KR 2077/2-3 and SFB/TR 15 ("Governance and the Efficiency of Economic Systems"), is gratefully acknowledged.

** Matthias Kräkel, Department of Economics, BWL II, University of Bonn, Adenauerallee 24-42, D-53113 Bonn, Germany, e-mail: m.kraekel@uni-bonn.de, phone: +49-228-739211, fax: +49-228-739210. 


\section{Introduction}

In rank-order tournaments, players compete for given prizes. The best performing player (e.g. the one with the highest output) receives the highest prize, the second best performer gets the second highest prize and so on. Distribution of prizes according to relative performance creates considerable incentives for all contestants since ex ante each player wants to be declared winner of the tournament. There are many examples for rank-order tournaments in practice: sales representatives compete for bonuses which have been fixed in advance (Murphy et al. 2004), workers take part in jobpromotion tournaments (Baker et al. 1994), athletes participate in sports contests (Szymanski 2003), lawyers compete in litigation contests (Wärneryd 2000), firms and individuals invest in external or internal rent-seeking contests (Gibbons 2005), managers receive relative performance pay (Gibbons and Murphy 1990), firms spend resources for advertising in winner-takeall markets (Schmalensee 1976), there are research tournaments (Schöttner 2007) and even tournaments in broiler production (Knoeber and Thurman 1994).

Theoretic models which analyze players' behavior in rank-order tournaments $^{1}$ typically focus on the effort or investment decision of contestants: The more input a player chooses relative to his opponents the higher will be his probability of winning the tournament. However, in real tournaments

\footnotetext{
${ }^{1}$ See, among many others, Lazear and Rosen (1981) and O'Keeffe et al. (1984). For a survey on tournaments and contests see Konrad (2007).
} 
players can often also decide on the risk of their behavior. For example, before firms choose their advertising expenditures, they can decide on whether introducing a new product (high risk) or not (low risk). In many tournaments, contestants first have the choice between using a standard technique (low risk) or switching to a new technique (high risk); thereafter they decide on effort or, more generally, on input to win the tournament.

This paper addresses such a two-stage tournament game with risk taking at the first stage and effort or input choice at the second stage. We focus on heterogeneous and risk averse workers so that interior pure-strategy equilibria at the effort stage are always asymmetric. This asymmetric outcome has important consequences on those effects which mainly determine risk taking in tournaments: First, the choice of risk influences the equilibrium efforts at the second stage (effort effect). Second, risk also influences the players' probabilities of winning (likelihood effect). Previous work on risk taking in two-stage tournaments has only considered symmetric equilibria at the effort stage 2. There, the effort effect and the likelihood effect were completely separate. However, this outcome will no longer hold, if players' utility functions at least slightly differ. Then both effort effect and likelihood effect are strictly interrelated which can be crucial for risk taking. In particular, we can show that in this situation the underdog (favorite), i.e. the player with the lower (higher) probability of winning in equilibrium, may prefer low risk (high risk) in order to maximize his winning probability which is impossible under symmetry. We also consider the role of risk aversion. Whereas 
under mean-variance preferences the more risk averse player always prefers maximum risk this result need not hold for alternative utility functions.

So far there is only a small number of papers which also address the problem of risk taking in two-stage tournaments. Hvide (2002) focuses on the case of homogeneous players. Since equilibrium efforts are always identical in his setting and the winning probability of each player is always one half irrespective of the risk level, basically there is no likelihood effect in the Hvide-model. Since equilibrium efforts are monotonically decreasing in risk, each player chooses maximum risk according to the effort effect so that players exert minimum effort at the second stage of the game. In the setting of Hvide (2002), maximizing risk at the first stage works like an implicit collusion for the effort choices at the second stage.

When introducing heterogeneity between the players, there are two possibilities in principle. Following O'Keeffe et al. (1984) we can differentiate between unfair and uneven tournaments. In an unfair two-person tournament, players choose identical efforts so that again we have a symmetric equilibrium like in the case of homogeneous contestants. However, one of the players has a lead and, hence, a higher probability of winning. In an uneven tournament, only asymmetric equilibria exist since players have different cost-of-effort functions or different preferences of winning (i.e. the subjective tournament prizes or the respective utilities of the players differ). Kräkel and Sliwka (2004) combine the problem of risk taking with unfair tournaments. In their setting, equilibria at the effort stage are always symmetric like in 
Hvide (2002). However, now both effort and likelihood effect are important. Whether the players prefer high or low risk in order to reduce effort costs depends on the magnitude of the favorite's lead. If the lead is small (large) both players are interested in choosing a high (low) risk in order to destroy overall incentives at the second stage according to the effort effect. Concerning the likelihood effect, there is an unambiguous result due to the symmetric equilibrium: The favorite (underdog) maximizes his winning probability by choosing low (high) risk. In this paper, we address risk taking by considering uneven tournaments in which only asymmetric interior equilibria can exist at the second stage. In this setting, it can be shown that the effort effect and the likelihood effect are strictly interrelated, which sharply contrasts with the findings for symmetric equilibria in Hvide (2002) and Kräkel and Sliwka (2004). This linkage between the two effects can lead to an equilibrium in which the underdog - and not the favorite - prefers low risk in order to maximize his winning probability.

There are some other papers that also deal with risk taking in tournaments. In the models by Gaba and Kalra (1999), Hvide and Kristiansen (2003) and Taylor (2003), players can solely decide on risk taking in the tournament; hence there is no effort effect and no possible linkage with the likelihood effect. Other papers analyze risk taking empirically. For example, Becker and Huselid (1992) consider individual behavior in stock-car racing. Their results show that drivers take more risk if tournament prizes and prize spreads are large. The findings of Brown et al. (1996) and Chevalier and Elli- 
son (1997) point out that presumable losers - contrary to presumable winners - prefer high risks in tournaments between mutual fund managers. Finally, the paper by Grund and Gürtler (2005) on professional soccer confirms the previous findings that leading players or teams (players that lie behind) prefer low-risk (high-risk) behavior. However, neither of the empirical papers addresses the effort effect.

The paper is organized as follows. The next section introduces the model. Section 3 considers the effort stage. Section 4 focuses on risk taking at the first stage of the game; it contains the main results. Section 5 concludes.

\section{The Model}

Two risk averse players $A$ and $B$ participate in a two-stage tournament. Player $i$ 's $(i=A, B)$ production or performance function is given by

$$
q_{i}=e_{i}+\varepsilon_{i}
$$

where $e_{i}$ denotes investment or effort chosen by player $i$. $\varepsilon_{A}$ and $\varepsilon_{B}$ are exogenous noise terms. The density of the composed random variable $\varepsilon_{j}-$ $\varepsilon_{i}(i, j=A, B ; i \neq j)$ is denoted by $g(\cdot)$ and the corresponding cumulative distribution function by $G(\cdot)$ which is assumed to be continuous and twice differentiable. The density $g(\cdot)$ is assumed to be symmetric around its unique mode at zero with variance $\sigma^{2}=\sigma_{i}^{2}+\sigma_{j}^{2}{ }^{2}$ For example, $\varepsilon_{i}$ and $\varepsilon_{j}$ may

\footnotetext{
${ }^{2}$ The assumption of a unimodal distribution is not unusual in tournament models; see, e.g., Drago et al. (1996), Hvide (2002), Chen (2003).
} 
be stochastically independent and normally distributed with $\varepsilon_{i} \sim N\left(\mu, \sigma_{i}^{2}\right)$ and $\varepsilon_{j} \sim N\left(\mu, \sigma_{j}^{2}\right)$. In that case, the convolution $g(\cdot)$ again is a normal distribution with $\varepsilon_{j}-\varepsilon_{i} \sim N\left(0, \sigma_{i}^{2}+\sigma_{j}^{2}\right)$.

Each player $i(i=A, B)$ has a strictly concave utility function $U_{i}$ which is separable in monetary income, $I_{i}$, and effort costs, $c\left(e_{i}\right)$ :

$$
U_{i}=u_{i}\left(I_{i}\right)-c\left(e_{i}\right)
$$

with $u_{i}^{\prime}\left(I_{i}\right)>0, u_{i}^{\prime \prime}\left(I_{i}\right)<0, \forall I_{i}$, and $c^{\prime}\left(e_{i}\right)>0, c^{\prime \prime}\left(e_{i}\right)>0, \forall e_{i}>0$, and $c(0)=0$. The utility functions are assumed to be common knowledge.

At the first stage of the tournament game (risk stage), both players $i$ and $j$ simultaneously choose their respective risks measured by the variances $\sigma_{i}^{2}$ and $\sigma_{j}^{2}$. At the second stage (effort stage), both players observe $\sigma_{i}^{2}$ and $\sigma_{j}^{2}$, and then simultaneously choose efforts $e_{i}$ and $e_{j}$. Effort choices together with the realizations of $\varepsilon_{i}$ and $\varepsilon_{j}$ determine $q_{i}$ and $q_{j}$ according to (1). If $q_{i}>$ $q_{j}$, player $i$ is declared winner of the tournament and receives the monetary prize $I_{i}=w_{1}$, whereas player $j$ 's monetary income is given by the loser prize $I_{j}=w_{2}<w_{1}(i, j \in\{A, B\} ; i \neq j)$.

\section{Optimal Effort Choices}

The subgame-perfect equilibrium of the two-stage game is derived by backward induction. First, the effort stage is considered where the players choose $e_{i}$ and $e_{j}$ in order to maximize their expected utilities $E U_{i}\left(e_{i}\right)$ and $E U_{j}\left(e_{j}\right)$, respectively, for given values of $\sigma_{i}^{2}$ and $\sigma_{j}^{2}$. Then we go backwards to the risk 
stage where the two players anticipate effort choices at stage $2, e_{i}^{*}\left(\sigma_{i}^{2}, \sigma_{j}^{2}\right)$ and $e_{j}^{*}\left(\sigma_{i}^{2}, \sigma_{j}^{2}\right)$, and choose their respective variances $\sigma_{i}^{2}$ and $\sigma_{j}^{2}$.

In stage 2 , player $i$ chooses $e_{i}$ to maximize

$$
E U_{i}\left(e_{i}\right)=u_{i}\left(w_{1}\right) G\left(e_{i}-e_{j}\right)+u_{i}\left(w_{2}\right)\left[1-G\left(e_{i}-e_{j}\right)\right]-c\left(e_{i}\right)
$$

with $G\left(e_{i}-e_{j}\right)=\operatorname{prob}\left\{q_{i}>q_{j}\right\}=\operatorname{prob}\left\{\varepsilon_{j}-\varepsilon_{i}<e_{i}-e_{j}\right\}$ denoting $i$ 's probability of winning. In analogy, player $j$ 's objective function can be written as

$$
E U_{j}\left(e_{j}\right)=u_{j}\left(w_{1}\right)\left[1-G\left(e_{i}-e_{j}\right)\right]+u_{j}\left(w_{2}\right) G\left(e_{i}-e_{j}\right)-c\left(e_{j}\right) .
$$

If an interior equilibrium solution in pure-strategies, $\left(e_{i}^{*}, e_{j}^{*}\right)$, exists at the effort stage, it will be characterized by the players' first-order conditions

$$
\begin{aligned}
\Delta u_{i} g\left(e_{i}^{*}-e_{j}^{*}\right)-c^{\prime}\left(e_{i}^{*}\right) & =0 \\
\Delta u_{j} g\left(e_{i}^{*}-e_{j}^{*}\right)-c^{\prime}\left(e_{j}^{*}\right) & =0
\end{aligned}
$$

with $\Delta u_{k}:=u_{k}\left(w_{1}\right)-u_{k}\left(w_{2}\right)(k=i, j)$ describing player $k$ 's additional utility from receiving more money in case of winning the tournament. Conditions (5) and (6) show that, in an interior pure-strategy equilibrium, the player with the larger utility spread $\Delta u_{k}$ exerts more effort. Of course, if luck becomes extremely large so that effort has not any real impact on the outcome of the tournament, an interior solution will not exist. Especially, if $\sigma^{2}=\infty$ the winner of the tournament is determined by pure luck, and each player's likelihood of winning is one half irrespective of the effort choices. Hence, in equilibrium both players choose zero efforts to minimize effort costs. 
As it is known in the tournament literature, pure-strategy equilibria will only exist if there is sufficient noise in the tournament and the players' cost function $c(\cdot)$ is sufficiently steep (Lazear and Rosen 1981, 845). In particular, strict concavity of the players' objective functions and, hence, existence of pure-strategy equilibria is assured if ${ }^{3}$

$$
\sup _{\sigma^{2}, \Delta e} \Delta u_{k} \cdot\left|g^{\prime}(\Delta e)\right|<\inf _{e>0} c^{\prime \prime}(e)
$$

with $\Delta e:=e_{i}-e_{j}$. Let $\sigma_{\min }^{2}$ denote the respective value of $\sigma^{2}$ at the left-hand side of (7), i.e. $\sigma_{\min }^{2}$ characterizes the minimum amount of risk necessary for assuring the existence of a pure-strategy equilibrium at the effort stage of the tournament game. We obtain the following results:

Proposition 1 If $c^{\prime}(0)=0$ and $\sigma_{\min }^{2} \leq \sigma^{2}<\infty$, there will exist a purestrategy equilibrium at the effort stage, $\left(e_{i}^{*}, e_{j}^{*}\right)$, being described by (5) and (6). Moreover, $\operatorname{sign}\left(\frac{\partial e_{i}^{*}}{\partial \sigma^{2}}\right)=\operatorname{sign}\left(\frac{\partial e_{j}^{*}}{\partial \sigma^{2}}\right)$.

Proof. The first part of the proposition has already been proved. To see that $\operatorname{sign}\left(\partial e_{i}^{*} / \partial \sigma^{2}\right)=\operatorname{sign}\left(\partial e_{j}^{*} / \partial \sigma^{2}\right)$ we have to implicitly differentiate the system of equations (5) and (6):

$$
\begin{aligned}
\frac{\partial e_{i}^{*}}{\partial \sigma^{2}} & =\frac{1}{|J|} \Delta u_{i} \frac{\partial g\left(e_{i}^{*}-e_{j}^{*}\right)}{\partial \sigma^{2}} \cdot c^{\prime \prime}\left(e_{j}^{*}\right) \\
\frac{\partial e_{j}^{*}}{\partial \sigma^{2}} & =\frac{1}{|J|} \Delta u_{j} \frac{\partial g\left(e_{i}^{*}-e_{j}^{*}\right)}{\partial \sigma^{2}} \cdot c^{\prime \prime}\left(e_{i}^{*}\right)
\end{aligned}
$$

with

$$
|J|:=E U_{i}^{\prime \prime}\left(e_{i}\right) E U_{j}^{\prime \prime}\left(e_{j}\right)+\Delta u_{i} \Delta u_{j}\left[g^{\prime}\left(e_{i}^{*}-e_{j}^{*}\right)\right]^{2}>0
$$

\footnotetext{
${ }^{3}$ For a similar condition see Schöttner (2007).
} 
denoting the Jacobian determinant. Comparing (8) and (9) yields

$$
\operatorname{sign}\left(\frac{\partial e_{i}^{*}}{\partial \sigma^{2}}\right)=\operatorname{sign}\left(\frac{\partial e_{j}^{*}}{\partial \sigma^{2}}\right)=\operatorname{sign}\left(\frac{\partial g\left(e_{i}^{*}-e_{j}^{*}\right)}{\partial \sigma^{2}}\right) .
$$

Proposition 1 shows that marginally increasing risk makes both players either increase or decrease efforts. From the first-order conditions (5) and (6), we know that if one player chooses more (less) effort, the other player will react in the same way (i.e. $\left.c^{\prime}\left(e_{i}^{*}\right) / \Delta u_{i}=c^{\prime}\left(e_{j}^{*}\right) / \Delta u_{j}\right)$. However, it is interesting to see in which situations both players increase or decrease their efforts. Increasing risk means that the probability mass under the density $g(\cdot)$ is shifted from the middle towards the tails of the distribution. If the effort difference $\left|e_{i}^{*}-e_{j}^{*}\right|$ is located near the mode, $\partial g\left(e_{i}^{*}-e_{j}^{*}\right) / \partial \sigma^{2}$ will be negative and the two players reduce their equilibrium efforts. If, on the contrary, $\left|e_{i}^{*}-e_{j}^{*}\right|$ is large and, hence, lies at one of the tails, then $\partial g\left(e_{i}^{*}-e_{j}^{*}\right) / \partial \sigma^{2}$ will be positive so that marginally increasing risk makes both players choose higher efforts in equilibrium. As an example consider $\varepsilon_{j}-\varepsilon_{i} \sim N\left(0, \sigma^{2}\right)$ which gives

$$
\frac{\partial g\left(e_{i}^{*}-e_{j}^{*}\right)}{\partial \sigma}=\frac{\exp \left\{-\frac{\left(e_{i}^{*}-e_{j}^{*}\right)^{2}}{2 \sigma^{2}}\right\}}{\sigma^{2} \sqrt{2 \pi}}\left(\frac{\left(e_{i}^{*}-e_{j}^{*}\right)^{2}}{\sigma^{2}}-1\right) .
$$

Thus, the derivative is positive (negative) if $\left|e_{i}^{*}-e_{j}^{*}\right|>(<) \sigma$. Note that the effort difference $e_{i}^{*}-e_{j}^{*}$ positively depends on the difference of the players' utility spreads, $\Delta u_{i}-\Delta u_{j}$, which yields the following corollary: 
Corollary 1 If $\left|\Delta u_{i}-\Delta u_{j}\right|$ is sufficiently large (small), an increase in risk $\sigma^{2}$ will result in larger (smaller) equilibrium efforts $\left(e_{i}^{*}, e_{j}^{*}\right)$.

The intuition for this result is the following: If the players are very heterogeneous in the sense that their utility spreads $\Delta u_{i}$ and $\Delta u_{j}$ differ significantly, then competition is highly uneven resulting in low effort levels of both players - the player with the very low utility spread (the underdog) is hardly motivated and exerts very little effort so that the other player (the favorite) also chooses a very low effort level as best response. However, increasing risk in this situation brings the poorly motivated underdog back into the race, since higher uncertainty works against the uneven competition. This effect makes both contestants exert higher effort levels. Consider now the opposite case. If there is only a small degree of heterogeneity (i.e. $\left|\Delta u_{i}-\Delta u_{j}\right|$ is small) in the tournament, competition will be rather even and efforts rather high. Increasing risk in such situation destroys incentives - the outcome of the tournament is rather determined by luck than by effort choice - so that equilibrium efforts fall.

\section{Optimal Risk Taking}

We differentiate between two situations. In the first scenario, each player can freely choose any variance which is equal or greater than $\sigma_{\min }^{2} / 2$. In the second scenario, each player can either choose low risk $\sigma_{L}^{2}$ or high risk $\sigma_{H}^{2}$ with $\sigma_{L}^{2}<\sigma_{H}^{2}$ and $2 \sigma_{L}^{2} \geq \sigma_{\min }^{2}$. 


\subsection{The Continuous Case}

In this subsection, player $i(j)$ chooses $\sigma_{i}^{2}\left(\sigma_{j}^{2}\right)$ at the first stage of the game with $\sigma_{i}^{2}, \sigma_{j}^{2} \geq \sigma_{\min }^{2} / 2$ anticipating that, at the second stage, efforts are chosen according to (5) and (6) in case of an interior solution, or that $e_{i}^{*}=e_{j}^{*}=0$ in case of $\sigma^{2}=\sigma_{i}^{2}+\sigma_{j}^{2}=\infty$. We obtain the following result:

Proposition 2 At the first stage, only corner or semi-corner solutions exist in which the player with the lower utility spread $\Delta u_{k}, k \in\{i, j\}$, chooses $\sigma_{k}^{2}=\infty$ as weakly dominant strategy.

Proof. The non-existence of interior solutions can be shown by contradiction. In case of an interior solution at the second stage, the players' objection functions at the first stage can be written s $^{4}$

$$
\begin{aligned}
& E U_{i}\left(\sigma_{i}^{2}\right)=u_{i}\left(w_{2}\right)+\Delta u_{i} \cdot G\left(e_{i}^{*}-e_{j}^{*} ; \sigma_{i}^{2}+\sigma_{j}^{2}\right)-c\left(e_{i}^{*}\right) \\
& E U_{j}\left(\sigma_{j}^{2}\right)=u_{j}\left(w_{2}\right)+\Delta u_{j} \cdot G\left(-\left[e_{i}^{*}-e_{j}^{*}\right] ; \sigma_{i}^{2}+\sigma_{j}^{2}\right)-c\left(e_{j}^{*}\right)
\end{aligned}
$$

with $G\left(\cdot ; \sigma_{i}^{2}+\sigma_{j}^{2}\right) \equiv G(\cdot)$, and $e_{i}^{*}=e_{i}^{*}\left(\sigma_{i}^{2}+\sigma_{j}^{2}\right)$ and $e_{j}^{*}=e_{j}^{*}\left(\sigma_{i}^{2}+\sigma_{j}^{2}\right)$ being described by (5) and (6). The first-order conditions yield

$$
\begin{aligned}
\Delta u_{i}\left(g\left(\Delta e^{*}\right)\left(\frac{\partial e_{i}^{*}}{\partial \sigma_{i}^{2}}-\frac{\partial e_{j}^{*}}{\partial \sigma_{i}^{2}}\right)+\frac{\partial G\left(\Delta e^{*}\right)}{\partial \sigma_{i}^{2}}\right) & =c^{\prime}\left(e_{i}^{*}\right) \frac{\partial e_{i}^{*}}{\partial \sigma_{i}^{2}} \\
\Delta u_{j}\left(-g\left(-\Delta e^{*}\right)\left(\frac{\partial e_{i}^{*}}{\partial \sigma_{j}^{2}}-\frac{\partial e_{j}^{*}}{\partial \sigma_{j}^{2}}\right)+\frac{\partial G\left(-\Delta e^{*}\right)}{\partial \sigma_{j}^{2}}\right) & =c^{\prime}\left(e_{j}^{*}\right) \frac{\partial e_{j}^{*}}{\partial \sigma_{j}^{2}}
\end{aligned}
$$

\footnotetext{
${ }^{4}$ Note that $G(-x)=1-G(x)$ because of the symmetry of the convolution.
} 
with $\Delta e^{*}:=e_{i}^{*}-e_{j}^{*}$. Since $g\left(\Delta e^{*}\right)=g\left(-\Delta e^{*}\right)$ (due to symmetry) and because of (5) and (6) (hence, the envelope theorem applies) we have that

$$
\begin{aligned}
g\left(\Delta e^{*}\right) \frac{\partial e_{j}^{*}}{\partial \sigma_{i}^{2}} & =\frac{\partial G\left(\Delta e^{*}\right)}{\partial \sigma_{i}^{2}} \\
\text { and } g\left(\Delta e^{*}\right) \frac{\partial e_{i}^{*}}{\partial \sigma_{j}^{2}} & =\frac{\partial G\left(-\Delta e^{*}\right)}{\partial \sigma_{j}^{2}} .
\end{aligned}
$$

However, since $\frac{\partial \sigma^{2}}{\partial \sigma_{i}^{2}}=\frac{\partial \sigma^{2}}{\partial \sigma_{j}^{2}}$ so that $\operatorname{sign}\left(\frac{\partial e_{j}^{*}}{\partial \sigma_{i}^{2}}\right)=\operatorname{sign}\left(\frac{\partial e_{i}^{*}}{\partial \sigma_{j}^{2}}\right)$ according to Proposition 1, and since $\operatorname{sign}\left(\frac{\partial G\left(\Delta e^{*}\right)}{\partial \sigma_{i}^{2}}\right) \neq \operatorname{sign}\left(\frac{\partial G\left(-\Delta e^{*}\right)}{\partial \sigma_{j}^{2}}\right)=\operatorname{sign}\left(\frac{\partial}{\partial \sigma_{j}^{2}}(1-\right.$ $\left.G\left(\Delta e^{*}\right)\right)$ ), equations (15) and (16) cannot hold at the same time.

Hence, at least on player chooses either $\sigma_{\min }^{2} / 2$ or $\infty$ in equilibrium. We can show that the player with the smaller utility spread always weakly prefers $\infty$. Let, w.l.o.g., $\Delta u_{i}>\Delta u_{j}$. If $i$ chooses $\sigma_{i}^{2}=\infty$, player $j$ will be indifferent between all possible values of $\sigma_{j}^{2}$ since $\sigma^{2}=\sigma_{i}^{2}+\sigma_{j}^{2}$. If $i$ chooses $\sigma_{i}^{2}<\infty$, player $j$ will either have

$$
\begin{aligned}
E U_{j}\left(\sigma_{j}^{2}<\infty\right) & =u_{j}\left(w_{2}\right)+\Delta u_{j} \cdot G\left(-\Delta e^{*}\right)-c\left(e_{j}^{*}\right) \\
\text { or } E U_{j}\left(\sigma_{j}^{2}=\infty\right) & =u_{j}\left(w_{2}\right)+\frac{\Delta u_{j}}{2}-c(0)
\end{aligned}
$$

with $\Delta e^{*}=e_{i}^{*}-e_{j}^{*}>0$ and $\left(e_{i}^{*}, e_{j}^{*}\right)$ being described by (5) and (6). Since $G\left(-\Delta e^{*}\right)<1 / 2$, we have $E U_{j}\left(\sigma_{j}^{2}=\infty\right)>E U_{j}\left(\sigma_{j}^{2}<\infty\right)$.

The intuition for the result of Proposition 2 is the following: The underdog, i.e. the player with the lower utility spread, has always an incentive to completely erase competition at the second stage by choosing infinitely large risk. Note that risk taking has two effects. First, it influences a player's effort choice and, hence, his effort costs (effort effect). Second, it determines 
the distribution $G(\cdot)$ and therefore a player's likelihood of winning (likelihood effect). For the underdog, both effects work into the same direction: By choosing infinite risk, effort incentives and effort costs are minimized. In addition, the underdog would exert less effort than the other player in case of an interior solution at the second stage and, therefore, would have a winning probability strictly smaller than one half. Infinitely high risk counterbalances the players' winning probabilities so that each one wins with probability one half.

\section{Example 1: Mean-variance preferences}

To analyze how the players' degree of risk aversion may influence their risk taking, let the utility function $u_{i}\left(I_{i}\right)$ in $(2)$ be further specified. We assume that both players have mean-variance preferences and hence a quadratic utility function ${ }^{5}$

$$
u_{k}\left(I_{k}\right)=I_{k}-r_{k} I_{k}^{2} \quad(k=i, j)
$$

with $r_{k}>0$ indicating player $k$ 's degree of risk aversion and $r_{k}<1 /\left(2 I_{k}\right), \forall I_{k}$, which guarantees a non-decreasing utility function. By using (17), the expected utilities of the two players at the second stage for given variances $\sigma_{i}^{2}$ and $\sigma_{j}^{2}$ can be written as

$$
\begin{aligned}
E U_{i}\left(e_{i}\right) & =E\left[I_{i}\right]-r_{i} E\left[I_{i}^{2}\right]-c\left(e_{i}\right) \\
& =w_{2}-r_{i} w_{2}^{2}+\left[\Delta w-r_{i}\left(w_{1}^{2}-w_{2}^{2}\right)\right] G(\Delta e)-c\left(e_{i}\right) \\
E U_{j}\left(e_{j}\right) & =w_{2}-r_{j} w_{2}^{2}+\left[\Delta w-r_{j}\left(w_{1}^{2}-w_{2}^{2}\right)\right] G(-\Delta e)-c\left(e_{j}\right)
\end{aligned}
$$

\footnotetext{
${ }^{5}$ See, e.g., Müller and Machina (1987).
} 
with $\Delta e=e_{i}-e_{j}$ and $\Delta w=w_{1}-w_{2}$. The first-order conditions for an interior solution at stage 2 are $^{6}$

$$
\begin{aligned}
& {\left[\Delta w-r_{i}\left(w_{1}^{2}-w_{2}^{2}\right)\right] g(\Delta e)=c^{\prime}\left(e_{i}\right)} \\
& {\left[\Delta w-r_{j}\left(w_{1}^{2}-w_{2}^{2}\right)\right] g(\Delta e)=c^{\prime}\left(e_{j}\right) .}
\end{aligned}
$$

By redefining $\Delta u_{k}:=\left[\Delta w-r_{k}\left(w_{1}^{2}-w_{2}^{2}\right)\right]$ and $u_{k}\left(w_{2}\right):=w_{2}-r_{k} w_{2}^{2}$ in the previous results, particularly in Propositions 1 and 2, we immediately get the following corollary:

Corollary 2 If the two players have mean-variance preferences according to (17), the player with the higher degree of risk aversion $r_{k}, k \in\{i, j\}$, will choose maximum risk $\sigma_{k}^{2}=\infty$ as weakly dominant strategy at stage 1 .

At first sight, it sounds counterintuitive that the more risk averse player unambiguously prefers to maximize risk. However, note that chosen risk is not identical with the players' income risks. Inspection of (18) and (19) gives the intuition for the finding of Corollary 2: The more risk averse player has the lower marginal return of winning, $\left[\Delta w-r_{k}\left(w_{1}^{2}-w_{2}^{2}\right)\right] g(\Delta e)(k \in\{i, j\})$, and therefore lower incentives at the second stage. ${ }^{7}$ Thus, he is the underdog who chooses maximum risk in order to minimize effort costs (effort effect) and to maximize his likelihood of winning (likelihood effect). However, note that the result of Corollary 2 need not hold for all possible utility functions

\footnotetext{
${ }^{6}$ Recall that $g(\Delta e)=g(-\Delta e)$ due to symmetry. Note that the terms in brackets are strictly positive because of the technical assumption $r_{k}<1 /\left(2 I_{k}\right), \forall I_{k}$, above.

${ }^{7}$ Note that player $i$ 's expected risk costs $E\left[r_{i} I_{i}^{2}\right]=r_{i}\left(w_{2}^{2}+\left(w_{1}^{2}-w_{2}^{2}\right) G(\Delta e)\right)$ monotonically increase in $i$ 's effort level. Analogous considerations hold for player $j$.
} 
as the player with the lower utility spread $\Delta u_{k}, k \in\{i, j\}$, is not necessarily the more risk averse individual.

\section{Example 2: Normally distributed noise and finite risk}

Let $\varepsilon_{j}-\varepsilon_{i} \sim N\left(0, \sigma_{i}^{2}+\sigma_{j}^{2}\right)$. To show that the underdog's preference for maximum risk crucially depends on the possibility of choosing infinitely high risk, in this example we assume that risk choice is limited to $\sigma_{\max }^{2} / 2$ for each player so that total maximum risk $\sigma^{2}=\sigma_{i}^{2}+\sigma_{j}^{2}=\sigma_{\max }^{2}$ still leads to an interior solution at the effort stage. ${ }^{8}$ Hence, at stage 1 , the players have to choose $\sigma_{i}^{2}$ and $\sigma_{j}^{2}$ from the interval $\left[\frac{\sigma_{\min }^{2}}{2}, \frac{\sigma_{\max }^{2}}{2}\right]$. To allow for explicit equilibrium solutions, let effort costs be exponential $c\left(e_{k}\right)=\exp \left\{c \cdot e_{k}\right\}-$ 1 with $c>0(k=i, j) .{ }^{9} \quad$ W.l.o.g., let player $i$ be the favorite and $j$ the underdog, i.e. $\Delta u_{i}>\Delta u_{j}$. Simple computations (see Appendix) show that, in stage 1 , we have

$$
\begin{aligned}
\frac{\partial E U_{i}}{\partial \sigma_{i}^{2}} & =\frac{\Delta u_{i}}{2 c \sigma^{3}}\left(1-\frac{\left(\Delta e^{*}\right)^{2}}{\sigma^{2}}-c \cdot \Delta e^{*}\right) \phi\left(\frac{\Delta e^{*}}{\sigma}\right) \\
\frac{\partial E U_{j}}{\partial \sigma_{j}^{2}} & =\frac{\Delta u_{j}}{2 c \sigma^{3}}\left(1-\frac{\left(\Delta e^{*}\right)^{2}}{\sigma^{2}}+c \cdot \Delta e^{*}\right) \phi\left(\frac{\Delta e^{*}}{\sigma}\right)
\end{aligned}
$$

with $\phi(\cdot)$ denoting the density of the standardized normal distribution and $\Delta e^{*}=\frac{1}{c} \ln \left(\frac{\Delta u_{i}}{\Delta u_{j}}\right)=$ const. Comparing (20) and (21) immediately shows that both derivatives cannot be zero at the same time. In both equations, the expression in parentheses is monotonically increasing in $\sigma^{2}$. Hence, if stationary

\footnotetext{
${ }^{8}$ The calculations in the appendix show that equilibrium efforts will only be positive for risk being not too large.

${ }^{9}$ Exponential cost functions are also used elsewhere in the literature. See, for example, Tadelis (2002), Kräkel and Sliwka (2004).
} 
points exist for $\sigma_{i}^{2}, \sigma_{j}^{2} \in\left[\frac{\sigma_{\min }^{2}}{2}, \frac{\sigma_{\max }^{2}}{2}\right]$, they will correspond to (local) minima so that we have strict corner solutions in the given setting. Interestingly, depending on the parameter values there may be an equilibrium in which both players prefer minimum risk: If, for example, $c \cdot \Delta e^{*}>1 \Leftrightarrow \ln \frac{\Delta u_{i}}{\Delta u_{j}}>1$, the favorite's objective function $E U_{i}\left(\sigma_{i}^{2}\right)$ is monotonically decreasing so that $i$ chooses $\sigma_{\min }^{2} / 2$ as a dominant strategy. Note that (21) has a unique root $\hat{\sigma}_{j}^{2}=\frac{\Delta e^{* 2}}{1+c \Delta e^{*}}-\sigma_{i}^{2}$. If $\hat{\sigma}_{j}^{2}>\sigma_{\max }^{2} / 2$ for $\sigma_{i}^{2}=\sigma_{\min }^{2} / 2$, then underdog $j$ will also choose minimum risk $\sigma_{j}^{2}=\sigma_{\min }^{2} / 2 .{ }^{10}$

To sum up, the discussion has shown that the finding of Hvide (2002) for homogeneous players that contestants prefer maximum risk does not in general apply to the case of heterogeneous players. The last example has shown that, on the contrary, both favorite and underdog may choose minimum risk in equilibrium. However, the most important topic - the interrelation of the effort effect and the likelihood effect - should be highlighted in more details in the next subsection.

\subsection{The Discrete Case}

In this subsection, two assumptions will be modified which do not seem to be very realistic. First, we skip the assumption that players can choose infinite risk. Second, now we assume that each player can only choose between two

\footnotetext{
${ }^{10} \mathrm{~A}$ necessary condition for this outcome is that $\frac{\Delta e^{* 2}}{1+c \Delta e^{*}}-\frac{\sigma_{\min }^{2}}{2}>\frac{\sigma_{\min }^{2}}{2} \Leftrightarrow \frac{\Delta e^{* 2}}{1+c \Delta e^{*}}>$ $\sigma_{\min }^{2}=\frac{\Delta u_{i} \exp \left\{-\frac{1}{2}\right\}}{c^{2} \sqrt{2 \pi}} \Leftrightarrow\left(\ln \left(\frac{\Delta u_{i}}{\Delta u_{j}}\right)\right)^{2}-\frac{\Delta u_{k} \exp \left\{-\frac{1}{2}\right\}}{\sqrt{2 \pi}}\left(1+\ln \left(\frac{\Delta u_{i}}{\Delta u_{j}}\right)\right)>0$ which can be satisfied.
} 
discrete risk levels - low risk and high risk. Both modifications are in line with the examples of Section 1 (e.g. new product introduction, stock-car racing). Moreover, part of the analysis of Hvide (2002) and the whole analysis of Kräkel and Sliwka (2004) deal with the discrete case and finite risk. Hence, it will be interesting to compare the case of heterogeneous players considered in this paper with the findings of the previous papers.

Both papers, Hvide (2002) and Kräkel and Sliwka (2004), focus on the case of symmetric equilibria at the effort stage 2. In Hvide (2002), players are assumed to be homogeneous which directly implies symmetry. In Kräkel and Sliwka (2004), players are heterogeneous in ability which yields a lead for the favorite; however, there are always symmetric equilibria at stage 2 since effort and ability are additively combined within each player's production function. Because of the symmetric equilibria at the effort stage, the effort effect and the likelihood effect are completely separate in both papers. This means that varying risk may influence both the equilibrium efforts and the winning probability, but the effort shifts have not any impact on the winning probability since in the symmetric equilibrium effort changes completely offset each other so that $\Delta e^{*}$ is zero. This specific characteristic simplifies the analysis a lot. In particular, equilibrium efforts can be explicitly calculated. ${ }^{11}$

\footnotetext{
${ }^{11}$ Note that in Example 2 above with normally distributed noise and exponential costs we also have explicit equilibrium efforts. However, this case is also very special since due to the cost function the difference of the equilibrium efforts, $\Delta e^{*}$, is constant; i.e. it does neither depend on risk $\sigma^{2}$ nor on the players' effort levels. Hence, in this special case the interrelation between effort and likelihood effect also disappears.
} 
Moreover, a player's expected utilities for different risk levels can be easily compared with each other. In this subsection, we would like to point out that with heterogeneous players and asymmetric equilibria at the effort stage, the effort effect and the likelihood effect will be strictly interrelated in general. This linkage can substantially change the risk behavior of players compared to the symmetric case.

Now, at stage 1 players $i$ and $j$ choose $\sigma_{i}^{2}, \sigma_{j}^{2} \in\left\{\sigma_{L}^{2}, \sigma_{H}^{2}\right\}$, respectively, with $\sigma^{2}=\sigma_{i}^{2}+\sigma_{j}^{2}$ ensuring an interior solution at the effort stage 2 described by the first-order conditions (5) and (6). Here, $\sigma_{L}^{2}$ denotes low risk, whereas $\sigma_{H}^{2}>\sigma_{L}^{2}$ stands for high risk. At stage 2 , the players exert efforts $e_{i}\left(\sigma^{2}\right)$ and $e_{j}\left(\sigma^{2}\right)$ for a given risk $\sigma^{2}$. We have to differentiate between three scenarios. First, there may be a high-risk situation in which both players choose $\sigma_{H}^{2}$ so that in equilibrium player $i$ 's winning probability (player $j$ 's probability of losing) is given by $G\left(\Delta e^{*}\left(2 \sigma_{H}^{2}\right) ; 2 \sigma_{H}^{2}\right)$ with $\Delta e^{*}\left(2 \sigma_{H}^{2}\right)=e_{i}^{*}\left(2 \sigma_{H}^{2}\right)-e_{j}^{*}\left(2 \sigma_{H}^{2}\right)$. Hence, choosing risk influences the shape of the cumulative distribution function, which exactly describes the likelihood effect in the symmetric cases of Hvide (2002) and Kräkel and Sliwka (2004). However, risk also influences each player's effort choice which normally results in an equilibrium effort difference that varies in $\sigma^{2}$ as indicated by $\Delta e^{*}\left(\sigma^{2}\right)$. Note that this is particularly true for the huge class of polynomial cost functions $c\left(e_{k}\right)=\frac{\gamma}{\delta+1} e_{k}^{\delta+1}$ $(k=i, j)$ with $\gamma>0$ and $\delta \geq 1$ which leads to

$$
\Delta e^{*}\left(\sigma^{2}\right)=\left(1-\left(\frac{\Delta u_{j}}{\Delta u_{i}}\right)^{\frac{1}{\delta}}\right) e_{i}^{*}\left(\sigma^{2}\right)
$$


since $c^{\prime}\left(e_{i}^{*}\right) / \Delta u_{i}=c^{\prime}\left(e_{j}^{*}\right) / \Delta u_{j}$ according to (5) and (6). As second scenario, there may be a low-risk situation in which both players choose $\sigma_{L}^{2}$ so that in equilibrium player $i$ 's winning probability (player $j$ 's probability of losing) can be written as $G\left(\Delta e^{*}\left(2 \sigma_{L}^{2}\right) ; 2 \sigma_{L}^{2}\right)$. Finally, there may be an intermediate situation with $G\left(\Delta e^{*}\left(\sigma_{L}^{2}+\sigma_{H}^{2}\right) ; \sigma_{L}^{2}+\sigma_{H}^{2}\right)$ in which one player chooses a low risk and the other one a high risk.

Of course, depending on the shape of the cumulative distribution function, the density and the cost function as well as the parameter values, different kinds of equilibria may be possible. However, the remainder of this subsection will concentrate on sufficient conditions for a low-risk equilibrium $\left(\sigma_{i}^{2}, \sigma_{j}^{2}\right)=$ $\left(\sigma_{L}^{2}, \sigma_{L}^{2}\right)$ at the first stage. Such selective proceeding seems to be appropriate for at least three reasons: (1) The results of the preceding subsection indicate that at least one player tend to prefer maximum risk (Proposition 2). Indeed, in the case of continuous and unlimited risk, at stage 2 players $i$ and $j$ always end up with dropping out of the competition by exerting zero effort since at least one player has chosen maximum risk as a weakly dominant strategy at stage 1. (2) Taking the main finding for homogeneous players of Hvide (2002) as a benchmark ("The unique equilibrium induces excessive risk taking and a low level of effort", p. 877), it is interesting to show that for heterogeneous players the opposite risk taking can be an equilibrium outcome. Moreover, on pp. 886-887 Hvide (2002) considers a (numerical) example with heterogeneous players and two discrete risk levels. There he demonstrates that under small heterogeneity both players choose a high risk, 
whereas under large heterogeneity the favorite chooses a low risk and the underdog a high risk. However, Hvide does not discuss the possibility of a low risk equilibrium $\left(\sigma_{L}^{2}, \sigma_{L}^{2}\right)$. (3) Finally, a detailed characterization of a low-risk equilibrium also indicates how other types of equilibria can be derived in analogy.

Let, w.l.o.g., $\Delta u_{i}>\Delta u_{j}$ so that player $i$ is the favorite and $j$ the underdog. From the discussion above we know that in this case $e_{i}^{*}\left(\sigma^{2}\right)>$ $e_{j}^{*}\left(\sigma^{2}\right), \forall \sigma^{2} ;$ hence $\Delta e^{*}\left(\sigma^{2}\right)>0$ and $G\left(\Delta e^{*}\left(\sigma^{2}\right) ; \sigma^{2}\right)>\frac{1}{2}>G\left(-\Delta e^{*}\left(\sigma^{2}\right) ; \sigma^{2}\right)$. Suppose that $\Delta u_{i}-\Delta u_{j}$ is sufficiently large so that increasing risk from $2 \sigma_{L}^{2}$ to $\sigma_{H}^{2}+\sigma_{L}^{2}$ would result in higher efforts and therefore also higher effort costs. If $2 \sigma_{L}^{2}$ and $\sigma_{H}^{2}+\sigma_{L}^{2}$ do not differ too much we can use the marginal analysis of Section 3, in particular Corollary 1, as an approximation which just shows this effect. We can also have a look at Figures 1 and 2 (see Appendix). Hence, concerning the effort effect neither player $i$ nor player $j$ has an incentive to deviate from $\left(\sigma_{i}^{2}, \sigma_{j}^{2}\right)=\left(\sigma_{L}^{2}, \sigma_{L}^{2}\right)$.

Now consider the likelihood effect. Graphically, increasing risk from $2 \sigma_{L}^{2}$ to $\sigma_{H}^{2}+\sigma_{L}^{2}$ means that the cumulative distribution function $G\left(\cdot ; \sigma^{2}\right)$ which at $\Delta e^{*}$ denotes $i$ 's winning probability "rotates clockwise" in the point $(0,1 / 2)$ (see Figures 1 and 2 in the Appendix). Note that in case of an asymmetric equilibrium at the effort stage 2, increasing risk has an additional effect on the players' winning probabilities: By increasing $e_{i}^{*}\left(\sigma^{2}\right)$ and $e_{j}^{*}\left(\sigma^{2}\right)$ it also influences $\Delta e^{*}\left(\sigma^{2}\right)$. Let $\Delta e^{*}\left(\sigma^{2}\right)$ rise from $\Delta e^{*}\left(2 \sigma_{L}^{2}\right)$ to $\Delta e^{*}\left(\sigma_{H}^{2}+\sigma_{L}^{2}\right)$ (recall that this will always happen in case of polynomial cost functions, see 
(22) with $\left.\Delta u_{i}>\Delta u_{j}\right)$.

Two different scenarios are possible: Either player $i$ 's winning probability increases and $j$ 's likelihood of winning decreases (as depicted by Figure 1) or, vice versa, $i$ 's winning probability decreases and $j$ 's winning probability increases (see Figure 2). If in the first scenario the effort effect dominates the likelihood effect for player $i$, or if in the second scenario the effort effect dominates the likelihood effect for player $j$, no one will deviate from $\left(\sigma_{i}^{2}, \sigma_{j}^{2}\right)=\left(\sigma_{L}^{2}, \sigma_{L}^{2}\right)$. It is important to emphasize that in the case of an unfair tournament with a symmetric equilibrium at stage 2 , as considered by Kräkel and Sliwka (2004), an increase (decrease) of the favorite's (underdog's) winning probability by increasing risk is impossible: There, $\Delta e^{*}\left(\sigma^{2}\right)=0$ and favorite $i$ 's winning probability in equilibrium is given by $G(\Delta a)$ with $\Delta a>0$ being a constant which denotes the favorite's lead. ${ }^{12}$ Hence, a "clockwise rotation" of $G\left(\cdot ; \sigma^{2}\right)$ would unambiguously decrease the favorite's likelihood of winning and increase the underdog's winning probability since it is always calculated at $\Delta a>0$.

Altogether, sufficient conditions for a low-risk equilibrium $\left(\sigma_{L}^{2}, \sigma_{L}^{2}\right)$ can be summarized as follows:

Proposition 3 Let $\Delta u_{i}-\Delta u_{j}$ be sufficiently large and $\Delta e^{*}\left(2 \sigma_{L}^{2}\right)<\Delta e^{*}\left(\sigma_{H}^{2}+\right.$

\footnotetext{
${ }^{12}$ This lead results from the ability difference between the two contestants in the unfair tournament. Each player $k(k=i, j)$ has an additive production function $q_{k}=e_{k}+\varepsilon_{k}+a_{k}$ with $e_{k}$ as endogenous effort, $\varepsilon_{k}$ as exogenous luck and $a_{k}$ as exogenous ability. Thus, $\Delta a:=a_{i}-a_{j}$ stands for favorite $i$ 's additional ability.
} 
$\left.\sigma_{L}^{2}\right)$ with $g\left(\Delta e^{*}\left(2 \sigma_{L}^{2}\right) ; 2 \sigma_{L}^{2}\right)<g\left(\Delta e^{*}\left(\sigma_{H}^{2}+\sigma_{L}^{2}\right) ; \sigma_{H}^{2}+\sigma_{L}^{2}\right)$.

If either (1) $G\left(-\Delta e^{*}\left(2 \sigma_{L}^{2}\right) ; 2 \sigma_{L}^{2}\right)>G\left(-\Delta e^{*}\left(\sigma_{H}^{2}+\sigma_{L}^{2}\right) ; \sigma_{H}^{2}+\sigma_{L}^{2}\right)$ and

$$
\begin{gathered}
\Delta u_{i}\left[G\left(\Delta e^{*}\left(\sigma_{H}^{2}+\sigma_{L}^{2}\right) ; \sigma_{H}^{2}+\sigma_{L}^{2}\right)-G\left(\Delta e^{*}\left(2 \sigma_{L}^{2}\right) ; 2 \sigma_{L}^{2}\right)\right] \leq \\
c\left(e_{i}^{*}\left(\sigma_{H}^{2}+\sigma_{L}^{2}\right)\right)-c\left(e_{i}^{*}\left(2 \sigma_{L}^{2}\right)\right),
\end{gathered}
$$

or (2) $G\left(\Delta e^{*}\left(2 \sigma_{L}^{2}\right) ; 2 \sigma_{L}^{2}\right)>G\left(\Delta e^{*}\left(\sigma_{H}^{2}+\sigma_{L}^{2}\right) ; \sigma_{H}^{2}+\sigma_{L}^{2}\right)$ and

$$
\begin{gathered}
\Delta u_{j}\left[G\left(-\Delta e^{*}\left(\sigma_{H}^{2}+\sigma_{L}^{2}\right) ; \sigma_{H}^{2}+\sigma_{L}^{2}\right)-G\left(-\Delta e^{*}\left(2 \sigma_{L}^{2}\right) ; 2 \sigma_{L}^{2}\right)\right] \leq \\
c\left(e_{j}^{*}\left(\sigma_{H}^{2}+\sigma_{L}^{2}\right)\right)-c\left(e_{j}^{*}\left(2 \sigma_{L}^{2}\right)\right),
\end{gathered}
$$

then $\left(\sigma_{i}^{2}, \sigma_{j}^{2}\right)=\left(\sigma_{L}^{2}, \sigma_{L}^{2}\right)$ will be an equilibrium.

Note that in case (1) both the effort effect and the likelihood effect work into the same direction for underdog $j$ so that he is not interested in choosing a high risk at all. This case should be highlighted since in the continuous case with unlimited risk (Subsection 4.1) the underdog always chooses maximum risk as a weakly dominant strategy. However, favorite $i$ faces a trade-off in case (1). On the one hand, deviating to high risk would increase his likelihood of winning; on the other hand, deviation would also increase effort costs. Hence, favorite $i$ also prefers not to deviate from $\left(\sigma_{L}^{2}, \sigma_{L}^{2}\right)$ if for him the effort effect dominates the likelihood effect (see condition (23)). Case (2) describes just the opposite scenario. Here, both the effort effect and the likelihood effect support a low-risk choice of favorite $i$ whereas underdog $j$ faces a trade-off (see condition (23)). 


\section{Conclusion}

In rank-order tournaments, players often can choose both risk and effort (or investment). Previous papers on risk taking in two-stage tournaments have focused on the case of symmetric equilibrium efforts at the second stage. The findings of this paper point out that switching to asymmetric behavior at the effort stage can change players' risk taking substantially.

In general, there are two effects that are decisive for a player's risk choice - an effort effect (i.e. more risk strengthens or weakens effort incentives) and a likelihood effect (i.e. more risk increases or decreases a player's winning probability). Whereas both players may be interested in the same risk choice in order to decrease total efforts and hence effort costs, their interests strictly differ concerning the likelihood effect since winning probabilities sum up to one.

The previous literature on risk taking has considered either rank-order tournaments with homogeneous players or so-called "unfair tournaments". In both cases, we always have a symmetric equilibrium at the effort stage which unambiguously separates the effort effect from the likelihood effect. However, if the players' utility functions at least slightly differ, only asymmetric pure-strategy equilibria can exist at the effort stage. The analysis of this paper shows that this asymmetry has important implications for players' risk taking since now effort effect and likelihood effect are strictly interrelated. In "unfair tournaments", the favorite (underdog) always prefers low (high) 
risk concerning the likelihood effect. If heterogeneous players compete within an "uneven tournament", these preferences may be exactly reverse. 


\section{Appendix}

Computation of (20) and (21):

An interior solution at stage $2,\left(e_{i}^{*}, e_{j}^{*}\right)$, is characterized by (5) and (6):

$$
\begin{aligned}
& \frac{\Delta u_{i}}{\sigma \sqrt{2 \pi}} \cdot \exp \left\{-\frac{\left(e_{i}^{*}-e_{j}^{*}\right)^{2}}{2 \sigma^{2}}\right\}=c \cdot \exp \left\{c \cdot e_{i}^{*}\right\} \\
& \frac{\Delta u_{j}}{\sigma \sqrt{2 \pi}} \cdot \exp \left\{-\frac{\left(e_{i}^{*}-e_{j}^{*}\right)^{2}}{2 \sigma^{2}}\right\}=c \cdot \exp \left\{c \cdot e_{j}^{*}\right\}
\end{aligned}
$$

with $\sigma=\sqrt{\sigma_{i}^{2}+\sigma_{j}^{2}}$ yielding $e_{k}^{*}=\frac{1}{c} \cdot \ln \left(\frac{\Delta u_{k}}{c \cdot \sigma} \phi\left(\frac{\Delta e^{*}}{\sigma}\right)\right) \quad(k=i, j)$

$(\phi(\cdot)$ denotes the density of the standardized normal distribution) and

$$
\Delta e^{*}=e_{i}^{*}-e_{j}^{*}=\frac{1}{c} \cdot \ln \left(\frac{\Delta u_{i}}{\Delta u_{j}}\right)
$$

Concerning the first stage, from (13)-(16) we know that

$$
\begin{aligned}
\frac{\partial E U_{i}}{\partial \sigma_{i}^{2}} & =\Delta u_{i}\left(-g\left(\Delta e^{*}\right) \frac{\partial e_{j}^{*}}{\partial \sigma_{i}^{2}}+\frac{\partial G\left(\Delta e^{*}\right)}{\partial \sigma_{i}^{2}}\right) \\
\frac{\partial E U_{j}}{\partial \sigma_{j}^{2}} & =\Delta u_{j}\left(-g\left(\Delta e^{*}\right) \frac{\partial e_{i}^{*}}{\partial \sigma_{j}^{2}}+\frac{\partial G\left(-\Delta e^{*}\right)}{\partial \sigma_{j}^{2}}\right) .
\end{aligned}
$$

In the case of normally distributed noise we obtain from (A3) and (A4)

$$
\frac{\partial e_{k}^{*}}{\partial \sigma_{l}^{2}}=-\frac{1}{2 c \sigma^{2}}+\frac{\left(\Delta e^{*}\right)^{2}}{2 c \sigma^{4}} \quad(k=i, j ; l=i, j) .
$$

Moreover, we have $g\left(\Delta e^{*}\right)=\frac{1}{\sigma} \phi\left(\frac{\Delta e^{*}}{\sigma}\right)$ and $G\left(\Delta e^{*}\right)=\Phi\left(\frac{\Delta e^{*}}{\sigma}\right)$ with $\frac{\partial G\left(\Delta e^{*}\right)}{\partial \sigma_{i}^{2}}=$ $\frac{-\Delta e^{*}}{2 \sigma^{3}} \phi\left(\frac{\Delta e^{*}}{\sigma}\right)(\Phi(\cdot)$ denotes the cdf of the standardized normal distribution). Similarly, we obtain $G\left(-\Delta e^{*}\right)=\Phi\left(\frac{-\Delta e^{*}}{\sigma}\right)$ with $\frac{\partial G\left(-\Delta e^{*}\right)}{\partial \sigma_{j}^{2}}=\frac{\Delta e^{*}}{2 \sigma^{3}} \phi\left(\frac{\Delta e^{*}}{\sigma}\right)$ $(\phi(x)=\phi(-x), \forall x)$. Putting all together, (A5) and (A6) can be rewritten as (20) and (21). 


\section{References}

Baker, G.P., Gibbs, M. and B. Holmstrom (1994): The Wage Policy of a Firm. Quarterly Journal of Economics 109, 921-955.

Becker, B.E. and M.A. Huselid (1992): The Incentive Effects of Tournament Compensation Systems. Administrative Science Quarterly 37, 336-350.

Brown, K.C., Harlow, W.V. and L.T. Starks (1996): Of Tournaments and Temptations: An Analysis of Managerial Incentives in the Mutual Fund Industry. Journal of Finance 51, 85-110.

Chen, K.-P. (2003): Sabotage in Promotion Tournaments. Journal of Law, Economics and Organization 19, 119-140.

Chevalier, J.A. and G.D. Ellison (1997): Risk Taking by Mutual Funds as a Response to Incentives, Journal of Political Economy 105, 1167-1200.

Drago, R., Garvey, G.T. and G.K. Turnbull (1996): A Collective Tournament. Economics Letters 50, 223-227.

Gaba, A. and A. Kalra (1999): Risk Behavior in Response to Quotas and Contests. Marketing Science 18, 417-434.

Gibbons, R. (2005): Four Formal(izable) Theories of the Firm?. Journal of Economic Behavior and Organization 58, 200-245. 
Gibbons, R. and K.L. Murphy (1990): Relative Performance Evaluation for Chief Executive Officers. Industrial and Labor Relations Review 43, 30-S-51-S.

Grund, C. and O. Gürtler (2005): An Empirical Study on Risk Taking in Tournaments. Applied Economics Letters 12, 457-461.

Hvide, H. (2002): Tournament Rewards and Risk Taking. Journal of Labor Economics 20, 877-898.

Hvide, H. and E.G. Kristiansen (2003): Risk Taking in Selection Contests. Games and Economic Behavior 42, 172-179.

Knoeber, C.R. and W.N. Thurman (1994): Testing the Theory of Tournaments: An Empirical Analysis of Broiler Production. Journal of Labor Economics 12, 155-179.

Konrad, K.A. (2007): Strategy in Contests - An Introduction. WZB Discussion Paper SP II 2007-01.

Kräkel, M. and D. Sliwka (2004): Risk Taking in Asymmetric Tournaments. German Economic Review 5, 103-116.

Lazear, E.P. and S. Rosen (1981): Rank-Order Tournaments as Optimum Labor Contracts. Journal of Political Economy 89, 841-864.

Müller, S.M. and M.J. Machina (1987): Moment Preferences and Polynomial Utility. Economics Letters 23, 349-353. 
Murphy, W.H., Dacin, P.A. and N.M. Ford (2004): Sales Contest Effectiveness: An Examination of Sales Contest Design Preferences of Field Sales Forces. Journal of the Academy of Marketing Science 32, 127143.

O’Keeffe, M., Viscusi, W.K. and R.J. Zeckhauser (1984): Economic Contests: Comparative Reward Schemes. Journal of Labor Economics 2, 27-56.

Schmalensee, R. (1976): A Model of Promotional Competition in Oligopoly. Review of Economic Studies 43, 493-507.

Schöttner, A. (2007): Fixed-Prize Tournaments versus First-Price Auctions in Innovation Contests. Economic Theory (forthcoming).

Szymanski, S. (2003): The Economic Design of Sporting Contests. Journal of Economic Literature 41, 1137-1187.

Tadelis, S. (2002): The Market for Reputations as an Incentive Mechanism. Journal of Political Economy 110, 854-882.

Taylor, J. (2003): Risk-Taking Behavior in Mutual Fund Tournaments. Journal of Economic Behavior and Organizations 50, 373-383.

Wärneryd, K. (2000): In Defense of Lawyers: Moral Hazard as an Aid to Cooperation. Games and Economic Behavior 33, 145-158. 


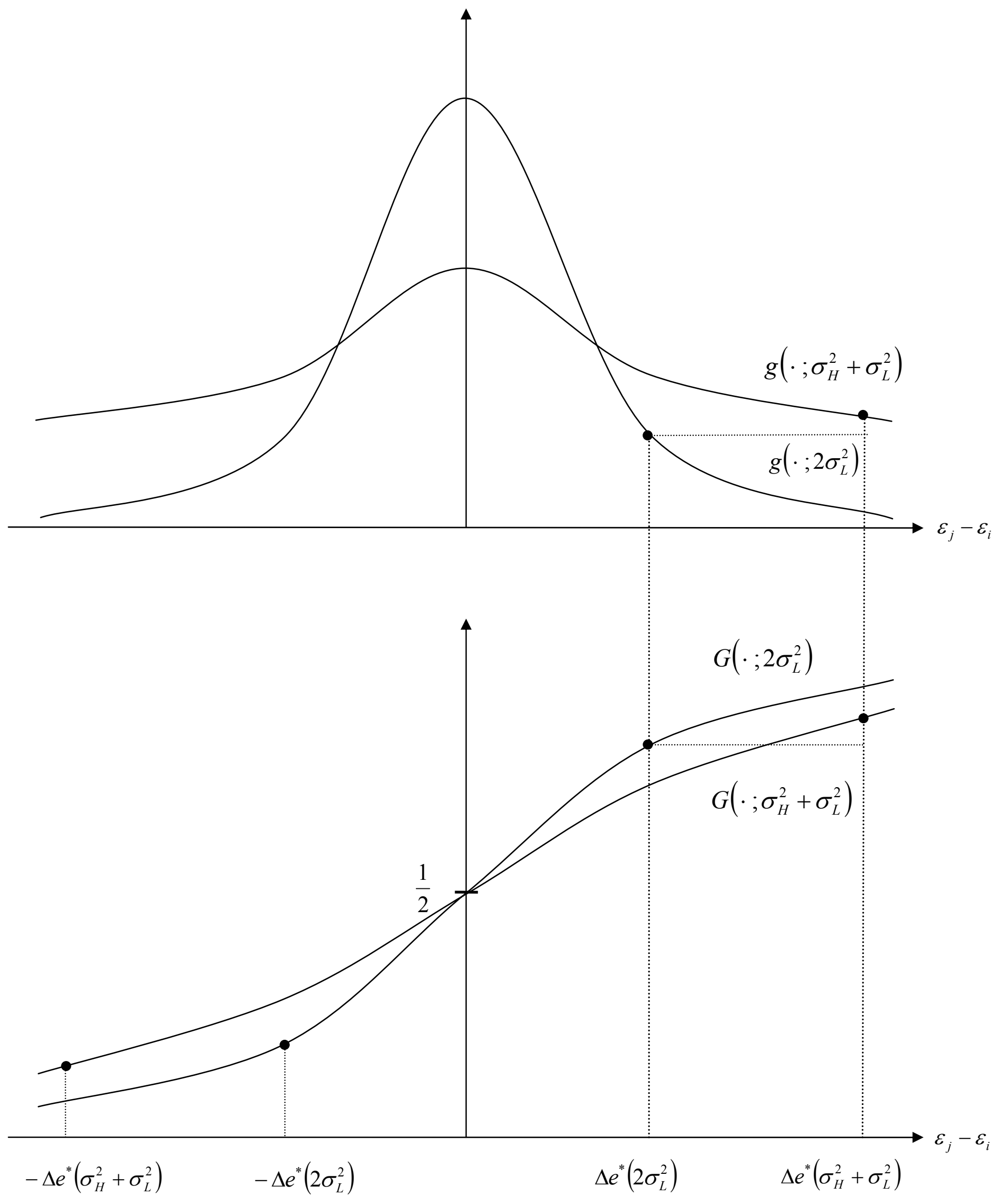

Figure 1: Underdog $j$ prefers low risk as a result of both effort effect and likelihood effect 


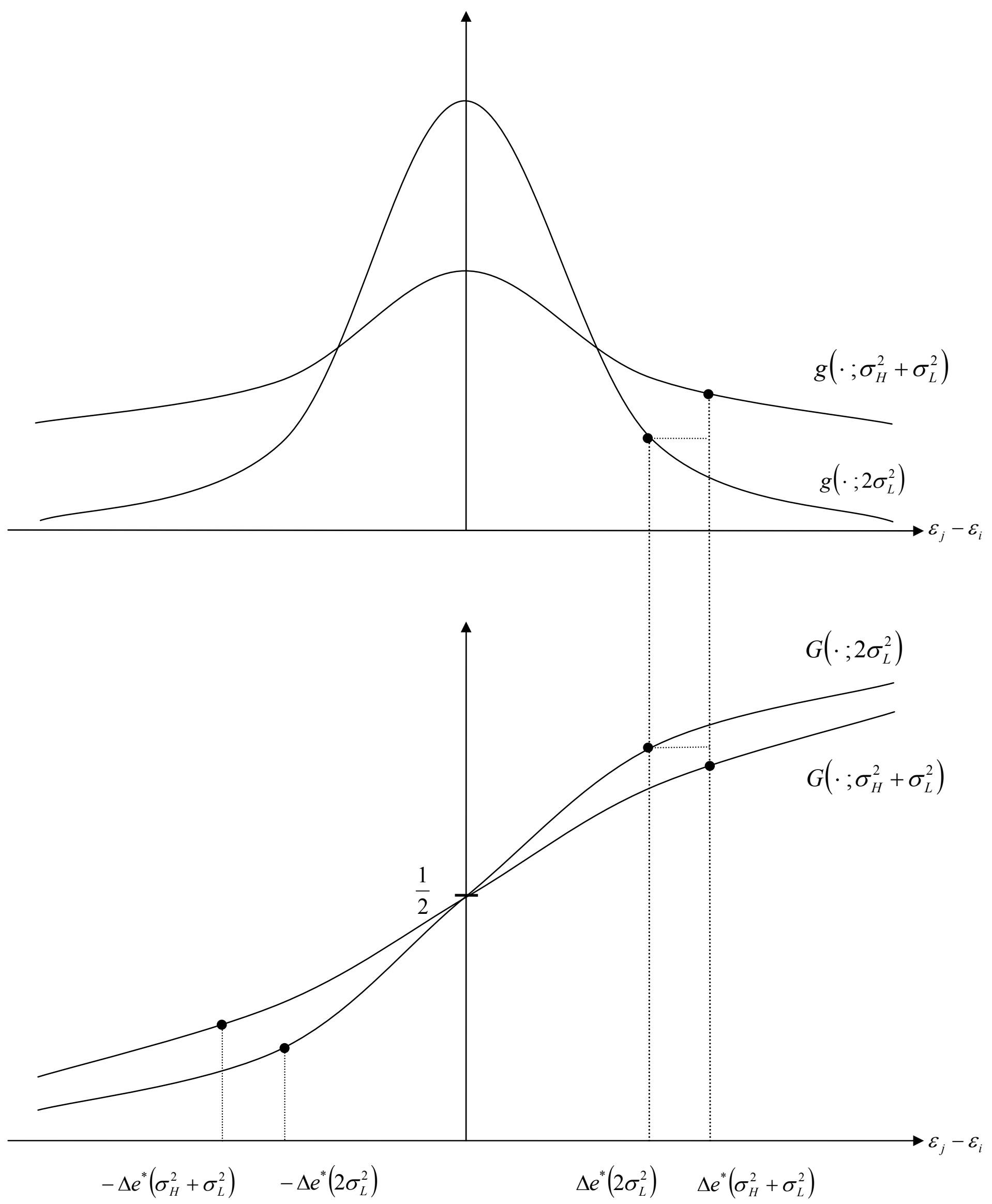

Figure 2: Favorite $i$ prefers low risk as a result of both effort effect and likelihood effect 